\title{
Enseignement du lexique et de la syntaxe
}

Un corpus multimédia comme point d'appui pour étudiants de niveau

$\mathrm{Bac}+5$ dans les métiers des industries des langues

Jean-Louis Vidalenc

\section{(2) OpenEdition}

\section{Journals}

Édition électronique

URL : http://journals.openedition.org/asp/2853

DOI : 10.4000/asp.2853

ISSN : 2108-6354

Éditeur

Groupe d'étude et de recherche en anglais de spécialité

\section{Édition imprimée}

Date de publication : 1 décembre 1998

Pagination : 247-260

ISSN : 1246-8185

\section{Référence électronique}

Jean-Louis Vidalenc, «Enseignement du lexique et de la syntaxe », ASp [En ligne], 19-22 | 1998, mis en ligne le 26 mars 2012, consulté le 20 avril 2019. URL : http://journals.openedition.org/asp/2853 ; DOI : $10.4000 /$ asp.2853

Ce document a été généré automatiquement le 20 avril 2019.

Tous droits réservés 


\section{Enseignement du lexique et de la syntaxe}

Un corpus multimédia comme point d'appui pour étudiants de niveau $\mathrm{Bac}+5$ dans les métiers des industries des langues

Jean-Louis Vidalenc

\section{Introduction}

1 Cet article se propose de présenter quelques éléments d'un dispositif pédagogique susceptible de contribuer à diversifier les débouchés professionnels des étudiants en anglais (que ce soit en filière traditionnelle, en LEA ou en Langue de Spécialités ${ }^{1}$ ). Ce projet prend en compte l'acquis de diverses préparations, trop nombreuses pour être recensés ici en détail, qui ont fait leurs preuves dans de nombreux établissements et qui débouchent sur des professions où une connaissance universitaire de l'anglais est prérequise (enseignant $\mathrm{du}$ primaire, du secondaire, du supérieur, traducteur [plus récemment terminologue], métiers du journalisme, chercheur scientifique, toutes les strates de secrétariat), etc. En retenant particulièrement l'apport de recherches récentes en civilisation américaine et britannique, en lexicologie et en syntaxe, en prenant appui sur nos propres travaux sur l'analyse linguistique de corpus d'histoire des sciences, d'anglais de la presse du cinéma et d'Internet, il est envisageable de construire une formation de type DESS susceptible de permettre à une vingtaine d'étudiants d'occuper des postes de travail dans la documentation multimédia.

2 Ce n'est pas parce que l'on peut parfois s'interroger sur le contenu pédagogique réel de l'apport du multimédia et d'Internet dans quelques établissements que les efforts d'innovation en didactique au quotidien doivent se priver de ces outils. C'est de l'articulation effective de l'enseignement avec la recherche en train de se faire que dépendra la crédibilité des transferts de technologie vers les salles de TD de l'enseignement de masse. On ne doit pas oublier que l'expérience de nombreuses universités américaines montre que l'intégration de ces nouvelles technologies non seulement résout des difficultés issues de modifications sociologiques des groupes 
d'étudiants mais est aussi susceptible de leur assurer une formation débouchant sur un emploi qualifié.

\subsection{Gisement d'emplois, qualification, formation à Bac+5}

3 Nous partons de deux constats simples : en premier lieu, les documents rédigés en anglais qui sont communiqués quotidiennement, via Internet, aux administrations, aux entreprises, etc. sont bien plus nombreux qu'il y a dix ans. Peu de personnes, à qui ces mêmes documents sont adressés ou qui en auraient l'utilité, ont la maîtrise de l'anglais adéquate pour les comprendre, les évaluer, les résumer ou les synthétiser, ne parlons pas de les traduire. Si certaines entreprises privilégient la sous-traitance de tout ce qui leur paraît extérieur à leur métier, d'autres préfèrent que des informations susceptibles de se révéler « sensibles » soient traitées par du personnel « maison ».

Le second constat est que seule une minorité d'étudiants de niveau Bac+4 est réellement à l'aise avec la compréhension, l'analyse et la traduction de ces mêmes documents. Trop peu d'étudiants ont été au-delà du minimum exigible pour un parcours universitaire et n'ont acquis ni la culture générale, ni la rapidité de compréhension de textes plus longs que les devoirs proposés. Sans entrer dans le détail des évolutions sociologiques qui expliquent vraisemblablement ce qui peut être vécu comme une anomalie, un pourcentage faible d'anciens étudiants de second cycle a effectivement la capacité de synthétiser ou de traduire rapidement et correctement des documents de plusieurs pages. civilisationniste qui leur demande de lire un journal anglais ou américain par semaine, d'un littéraire qui leur suggère de lire un livre non cité dans le Guide des études, d'un spécialiste de l'oral qui leur conseille de « décortiquer » un film² récent, d'un linguiste qui souhaiterait qu'ils se constituent un petit corpus, illustrent ce décalage entre compétences souhaitables et compétences réelles.

6 Résorber l'écart entre les compétences acquises dans un enseignement de licence de plus en plus massifié et les exigences de nouveaux postes de travail amène à repenser l'enseignement en maîtrise et en DESS et à s'interroger sur l'articulation Bac+4, Bac+5.

7 Même si la diversité extrême des documents disponibles sur les nouveaux médias peut paraître mettre en difficulté les structures universitaires traditionnelles, les équipes de recherche qui s'intéressent à la rénovation pédagogique de l'enseignement supérieur disposent des moyens de répondre à cette nouvelle demande de formation.

8 Trois paliers de réponse sont envisageables. En premier, il apparaît vraisemblable que ce sont les étudiants qui auront le bagage le plus complet en langue et civilisations anglosaxonnes qui seront le plus à l'aise sur les nouveaux postes de travail. En second, les entreprises qui avaient à nourrir des sites sur la toile ont fait appel à du personnel formé aux métiers du journalisme et de la traduction, domaines dans lesquels l'université dispose d'un acquis. En troisième, une partie des documents diffusés sur Internet est immédiatement intégrée à des corpus de grande taille ou de taille plus réduite qui sont l'objet d'études universitaires.

9 C'est sur ce front de la constitution et de l'analyse de corpus correspondants aux besoins sociaux que se déterminera vraisemblablement la capacité des établissements d'enseignement supérieur à trouver des débouchés pour leurs étudiants dans les industries de la langue. 


\subsection{Trois volets complémentaires, constitution de corpus adaptés, analyse syntaxique, analyse lexicale}

10 Le dispositif d'enseignement qui nous paraît susceptible de préparer des étudiants francophones à la compréhension, la synthèse et la gestion, la traduction de documents multimédia est précisément construit sur l'analyse lexicale et syntaxique d'un panier de corpus de taille diverse.

11 Dans cet article, nous présenterons une des stratégies envisageables pour répondre à ce défi. Nous nous intéresserons en premier lieu à la complémentarité des grands corpus saisis en amont des dictionnaires monolingues ou bilingues et de corpus plus ciblés. En second, nous approfondirons la réflexion sur la constitution d'un panier de petits corpus adaptés à un objectif didactique. Enfin, nous illustrerons, par quelques exemples, comment des acquis récents en études de la syntaxe et du lexique peuvent résoudre des obstacles qui sont apparus dans la compréhension de documents multimédia.

Un aspect positif du projet est que cet enseignement et la recherche qui lui serait associée, adapté à la compréhension d'articles de presse, de films et des informations diffusées par Internet pourrait ouvrir une piste de diversification des études anglaises ; on sait que les évolutions sociologiques font que de bons étudiants n'ont pas le profil souhaitable pour exercer actuellement dans le second degré.

\section{L'emploi de grands corpus et de petits corpus ciblés est complémentaire}

13 Les anciens du GERAS des années 1984-1988 ont en mémoire les interventions de John Sinclair et d'Antoinette Renouf, lorsqu'ils ont présenté la recherche qui devait déboucher sur la série des dictionnaires Cobuild. L'analyse informatique des très grands corpus a totalement renouvelé la conception des dictionnaires et multiplié les services qu'ils sont susceptibles de rendre, tant aux anglicistes à plein temps qu'à ceux qui ont des besoins plus ponctuels de références lexicales.

L'apport de ces bases s'est révélé très précieux non seulement pour la recherche fondamentale en terminologie et pour satisfaire des curiosités d'enseignants que les dictionnaires antérieurs laissaient sur leur faim, mais pour surtout pour répondre à des questions de pédagogie quotidienne. Pour traduire dégraissage ou licenciement collectif en anglais, il peut être utile d'avoir accès à la distribution sur six mois ${ }^{3}$ dans The Independent de delayering, delevelling, downsizing, flattening. La courbe de fréquence de zippergate de décembre 1997 à mars 1998 ne revêt pas qu'un intérêt anecdotique. Lorsque l'enseignant de civilisation ou de traduction choisit de travailler en TD sur un article de journal où backbenchery fait irruption, il est utile de pouvoir vérifier que cette lexie traduisible par un chahut parlementaire n'est apparue qu'une fois en six mois.

Les grands corpus que ce soient les plus anciens comme Brown et LOB, ou les plus récents comme ceux utilisés par les équipes de recherche lexicographique, ont fait leurs preuves depuis bien des années. On doit rappeler que l'approche inverse qui consiste à inventorier les termes correspondant aux besoins d'un métier ou du commerce existe depuis au moins 26 siècles. La quasi-totalité des métiers ou des industries a besoin à un moment 
donné d'un inventaire bilingue ou multilingue des termes employés dans l'exercice de son activité.

Cette demande sociale recoupe la pratique des écoles de traducteurs et ce qu'il est envisageable de réaliser comme recherche en un temps limité. Parmi les mémoires de ces écoles, on notera des microdomaines où trente termes sont recensés ${ }^{4}$.

Des bases de données terminologiques ${ }^{5}$ d'une autre ampleur s'avèrent nécessaires pour répondre à des besoins industriels plus conséquents. L'écart entre 30 et 14000 termes peut paraître important, mais on doit retenir ici la similitude de la démarche : dans les deux cas, on est parti d'un corpus correspondant à une activité professionnelle.

L'exemple des mémoires de fin d'études d'école de traduction montre d'ailleurs que ce ciblage sur la pratique langagière d'un métier représente un point d'appui solide pour l'apprentissage de la lexicologie. L'étudiant peut proposer une solution de traduction après avoir pris en compte les revues professionnelles et discuté avec les praticiens.

Dans les domaines scientifiques, notre proposition de prendre comme corpus de départ la bibliographie d'un DEA permet de disposer de microcorpus bien identifiés. Non seulement cela facilite la consultation des spécialistes de la question, mais cela permet aussi des regroupements macrodisciplinaires.

L'enseignement au quotidien doit d'ailleurs encourager à la modestie en ce qui concerne la taille des corpus; si on met bout à bout les textes qu'il est possible de traiter dans un TD annuel de traduction, de rédaction ou de synthèse de textes, on aboutit à un ensemble de documents moins volumineux que la quasi-totalité des corpus disponibles. La critique de certains doit être relativisée : l'expérience des formations rodées à la traduction ou à la correspondance de presse montre d'ailleurs que c'est en travaillant sur ces corpus restreints que les étudiants ont fait les progrès leur permettant d'accomplir les tâches langagières qu'ils ont à effectuer.

21 La pratique de l'enseignement montre donc qu'il y a plus complémentarité qu'opposition entre grands et petits corpus, l'étudiant doit pouvoir disposer des uns et des autres au long de son parcours même si c'est évidemment avec son propre corpus qu'il pourra acquérir une autonomie indispensable.

\section{Constitution d'une palette de corpus}

\subsection{L'acquis antérieur}

22 Notre expérience de la constitution et du renouvellement ${ }^{6}$ des corpus en amont d'une tâche d'enseignement nous a amené à privilégier une stratégie de recherche et d'enseignement où les sources documentaires étaient sélectionnées en fonction d'une tâche didactique précise. L'expérience de confrontation avec des corpus différents, la réflexion sur ces corpus avant que l'accès à Internet ne soit généralisé nous ont permis de mieux gérer les limites inévitables de tout corpus ou ensemble de corpus.

Lorsque nous exercions dans une université scientifique, nous avons à côté de deux corpus indépendants d'articles de recherche et de manuels constitué un corpus d'oeuvres littéraires, afin de disposer de sources plus proches de celles utilisées par les auteurs de grammaire récente. Nous avons par ailleurs dès 1984 commencé la constitution d'un micro corpus de scénarios de films pour disposer d'une source semi-orale. 

$1965^{7}$ encourage à bien délimiter la source de documents essentiels et à éviter d'en être prisonnier. L'histoire récente des applications de la linguistique à l'enseignement compte quelques dérapages notoires, conséquence d'un choix de corpus ne tenant pas compte de l'expérience collective accumulée au fil des ans. Ainsi il a pu être dit dans un atelier de la SAES du congrès de Lyon en 1981, qu'il n'y avait pas de modaux dans la langue des scientifiques. De même on a pu soutenir dans une réunion de travail d'une équipe CNRS qu'il n'y avait pas de have+en dans les textes de biologie etc. Plus fondamentalement, il apparait crucial d'encourager les étudiants à se constituer leur propre microcorpus indépendamment de ce qui peut être effectué comme travail en TD, à acquérir l'initiative et l'autonomie qui se révéleront indispensables sur leur poste de travail.

\subsection{Corpus saisis sur Internet}

作 besoins de différents postes de travail susceptibles d'intéresser les étudiants, est telle que même un échantillonnage très diversifié de textes sera inévitablement peu représentatif.

Il nous est apparu important de constituer en quatrième année les bases d'une approximation raisonnable. L'hypothèse de travail retenue est que la consultation régulière de serveurs bien identifiés permettra de constituer les bases d'un enseignement à Bac+5.

29 Le premier corpus constitué a été un corpus d'articles de presse, l'objectif a été de recueillir sur disquettes - permettant la consultation par les étudiants hors du cours des analyses de journalistes différents sur des thèmes d'actualité. Un grand nombre de journaux est accessible sur le réseau ${ }^{8}$. Toutefois les restrictions d'accès pratiqués par certains, l'insertion d'encarts publicitaires parasites, quelques difficultés de connexion, les exigences d'une didactisation du contenu en amont du TD amènent à stocker sur sauvegarde les textes et à ne pas trop travailler en direct ${ }^{9}$. Cela permet aussi de regrouper les documents par thème civilisationnel (politique générale, politique de l'environnement, politique universitaire, politique de santé, question irlandaise, etc.).

L'objectif pédagogique est que l'étudiant soit à l'aise - tant du point de vue de la langue que de la civilisation - avec la lecture des journaux de qualité. C'est par des études régulières de la presse récente que l'on pourra éviter les remarques acerbes - mais justifiées - de cabinets de recrutement déplorant l'insuffisance de la culture politique anglo-saxonne de nombreux diplômés de l'université.

31 Nous avons constitué cette année, pour le niveau $\mathrm{Bac}+4 \mathrm{Bac}+5$, un petit corpus pédagogique sur disquettes d'une vingtaine de textes saisis sur Internet de "science multidisciplinaire ». On connaît depuis 1965 les difficultés d'adéquation de la vulgarisation scientifique ${ }^{10}$ à l'enseignement, il s'agit de courts articles traitant de sujets 
scientifiques d'actualité (éthique du clonage humain, recherches sur le SIDA, la maladie de la vache folle, la malaria), ou plus permanents (l'extinction des dinosaures) intéressant des chercheurs de diverses disciplines (médecine, biologie, gestion des entreprises, géographie humaine, civilisation, etc.). Un aspect intéressant de ces documents dépouillés initialement pour une formation en traduction spécialisée, utilisés avec des étudiants de licence, maîtrise et doctorat.

Le deuxième corpus, plus adapté à la traduction spécialisée, a rassemblé divers documents que l'on pourrait appeler "science transdisciplinaire». Il s'agit de communiqués d'institutions de recherche et d'articles diffusés sur la toile traitant de sujets comme la vache folle, les réticences de grands laboratoires pharmaceutiques à faire des recherches de médicaments pour les populations non solvables, le clonage humain, la couche d'ozone dans l'atmosphère, les difficultés des lycées américains à avoir des programmes conséquents en mathématique et en biologie, l'extinction des dinosaures etc.

Ce corpus compte beaucoup de contributions de chercheurs d'un domaine conçues pour les chercheurs d'autres disciplines. Cette strate de langue sur laquelle nous travaillons depuis 1982 présente l'avantage de ne pas être opaque pour les non-spécialistes tout en ne souffrant pas des défauts de la vulgarisation pour bibliothèque de gare. Du point de vue de la langue, ces textes sont souvent bien écrits et permettent du fait de la présence de termes très spécialisés de servir de point d'appui à un enseignement de la terminologie.

Le troisième corpus a été constitué au départ pour fournir la documentation en amont d'un enseignement de traduction à une maîtrise LEA affaires et commerce. Il est construit autour d'un jumelage virtuel ${ }^{11}$, à partir d'une exploitation de son site Internet, avec une école de commerce américaine de la banlieue résidentielle de Chicago. Nous avons téléchargé quelques polycopiés, des fiches de lecture rédigées par des enseignants et des étudiants, nous y avons joint quelques conférences d'invités (dont une de Bill Gates). Nous avons aussi récupéré des documents analogues dans deux écoles de commerce de Nouvelle Angleterre. Surtout, le journal des étudiants s'est révélé une mine, tant pour les phénomènes de langue que pour la sociologie nord-américaine: états d'âmes des étudiants devant le stress de la sélection, de la pression des études, préparation des tests d'embauche, chroniques de la réussite professionnelle d'anciens étudiants, interviews du Doyen, de la secrétaire responsable des affectations dans les TD, compte rendu de la soirée étudiante au casino, de l'aide aux populations en difficulté aux environs du campus, de la critique gastronomique de restaurants en ville etc.

L'objectif premier de ce corpus est de sensibiliser les étudiants au mode de pensée et à l'expression orale et écrite de certains de leurs futurs interlocuteurs professionnels. On s'aperçoit toutefois que ce corpus a son intérêt pour l'étude de phénomènes linguistiques. C'est dans ce corpus que nous avons relevé he's $404^{12}$ dont nous parlerons plus loin.

Quelques difficultés techniques ont retardé la constitution d'un corpus informatisé d'histoire des sciences et nous ont contraint à nous limiter à la version papier de 1995. Ces textes, où des scientifiques de renom s'interrogent sur leur pratique, où des philosophes et des historiens des sciences réfléchissent sur cet acquis, représentent une bonne base pour amener des étudiants de formation littéraire à la compréhension de documents écrits par des chercheurs de disciplines très diverses. Ils constituent une source de documents plus riches en difficultés de traduction que des comptes rendus bien 
carrés d'expérience de laboratoires. Les repérages temporels précis procurent des éléments fiables pour l'initiation à la terminologie d'une discipline scientifique.

Le corpus le plus important en taille - il serait actuellement de l'ordre de douze mégas est un corpus virtuel ${ }^{13}$. Il s'agit d'une liste d'environ cinquante adresses $w^{\prime} w w^{14}$ où chacune correspond à un site où un scénario de film peut être consulté. De nombreuses associations d'étudiants - souvent allemandes, canadiennes ou australiennes - disposent de sauvegardes sur zip ou cd-roms, mais la simple connexion par modem ${ }^{15}$ permet de disposer de l'équivalent en taille de petits corpus électroniques.

En discutant avec des étudiants de maîtrise (ou de DESS d'autres établissements), il est apparu que cet ensemble de scénarios disponible pouvait servir de point d'appui à des corpus adaptés à la recherche que des étudiants peuvent mener à ce niveau. Par exemple, cela permettait d'observer les écarts entre langue normée et langue sous standard ou de cerner, sur étude de cas, l'emploi de la langue comme moyen de discrimination sociale ${ }^{16}$.

Cette complémentarité des corpus permet à l'étudiant de ne pas être prisonnier d'une seule source documentaire. On doit se rappeler que les incertitudes sur les débouchés font qu'il semble raisonnable de couvrir le plus large spectre possible de strates et de lexiques spécifiques d'anglais contemporain, de considérer chaque microcorpus, non comme une fin en soi mais comme un point d'appui assurant à l'étudiant la capacité de se débrouiller avec diverses sources de documentation. Autant il est utopique de vouloir englober dans une formation la totalité des phénomènes langagiers susceptibles d'être rencontrés par un internaute, autant il est envisageable de se constituer une méthode d'approche de quelques corpus diversifiés qui sera transposable aux autres ensembles de documents rencontrés lors d'un parcours professionnel.

\section{Grammaire et terminologie}

\subsection{Grammaire de la nouvelle ou grammaire d'ensemble de la langue}

Les exigences de la préparation des concours de second degré ont concentré les efforts des spécialistes de grammaires sur l'analyse grammaticale de textes susceptibles de se trouver au programme ou à l'oral des concours. Les textes journalistiques, proches à bien des égards de la grande majorité des textes que l'on rencontre sur la toile sont, dans l'ensemble, moins analysés qu'il y a une trentaine d'années.

41 Si l'on excepte la capacité connue de beaucoup de grands écrivains à pousser la langue dans ses retranchements et à faire fonctionner "à la limite» bien des marqueurs linguistiques, l'idée selon laquelle la langue non littéraire serait justifiable d'une analyse linguistique moins poussée que celle de la langue littéraire nous semble particulièrement pernicieuse.

\subsubsection{Premier exemple}

42 Le premier exemple que nous citerons est emprunté à la thèse de Claude Delmas (1987). Il mentionne cet emploi anglais du présent historique dans les légendes de photos du type

The Prince opens the fair yesterday 
la sensibilité langagière de beaucoup d'anglophones ${ }^{17}$ soit heurtée par des énoncés de ce type est un fait, qu'on les rencontre dans la presse ne peut être contesté et il faut en rendre compte. Nous rencontrons dans notre corpus Internet de science transdisciplinaire, au fil d'une rétrospective sur la maladie de la vache folle :

1989 : John Gummer, at the time the Minister for Agriculture, tries to give his daughter a beefburger in front of the cameras outside parliament (she refused).

La collision entre une date de l'histoire politique bien établie, le $\mathbf{s}$ de troisième personne singulier du présent de tries et le ed de refused nous semble plus heurter la règle d'usage que les exemples cités par Claude Delmas même si on peut y déceler la même logique de fonctionnement. Dans ces énoncés reprenant des manchettes de journal, la photo pouvait situer l'événement dans l'instant de perception du lecteur, dans l'énoncé saisi sur la toile, écrit par un ancien d'une école de journalisme, il n'y a pas de photo, mais le lecteur est inévitablement interpellé par la proximité de 1989 et de tries. On remarquera toutefois que le rédacteur invite le lecteur à se remémorer une séquence d'actualité télévisée. On observera que l'écrit supporte une suspension de la règle qui veut que ed corresponde à un repère sur une échelle temporelle externe à l'énonciateur. On notera toutefois que se vérifie ici la règle de bon sens mise en évidence par Paul Larreya depuis plusieurs années, avec le présent la vérité de la prédication est observable au moment de l'énonciation.

\subsubsection{Anaphore}

On sait que la maitrise de l'anaphore implicite, qui gouverne la présence de l'article défini (Hawkins 1978), se révèle un point délicat de l'apprentissage de la grammaire de l'anglais. Dans l'exemple suivant, relevé dans notre corpus de jumelage virtuel, nous rencontrons :

From day one at Kellogg we are told to keep perspective, to remember why we are here and not to get caught in the frenzy.

La présence de the devant frenzy s'explique parce que pour le rédacteur en chef du journal d'étudiants, comme pour ses lecteurs frenzy fait partie du programme sémique implicite de leur école. Cet exemple nous semble représentatif des très nombreux énoncés où l'explication d'un fait de langue passe par la prise en compte de données civilisationnelles.

\subsubsection{Subjonctif}

Avec certains emplois du subjonctif ${ }^{18}$, on peut observer un autre exemple où la langue des journalistes reconvertis dans l'écriture sur le net heurte la conscience de leur langue maternelle de beaucoup d'anglophones :

The German Government decided that it would not accept British beef as food in their country because of the risk that it potentially had to their population. Gummer was furious and demanded that less strict laws be taken through the EC Agriculture Committee.

According to Havens, U.S. growers have refused to meet Mexican requirements that cherries be fumigated for pests that are already prevalent 
in Mexico or where the cherry is not even host to the pest required to be eradicated.

La question essentielle ici n'est pas de trancher entre les linguistes qui comme André Joly privilégient l'analyse de be comme un subjonctif et ceux qui préfèrent parler de « trace de non-assertion » (Delmas et al. 1993 : 165). Elle est de constater que les textes largement diffusés comportent des formes dont l'explication ne fait pas l'objet d'un consensus. Certes, nous avons formé suffisamment de traducteurs au fil des ans pour savoir que l'on peut traduire correctement cet énoncé sans maitriser toutes les formes grammaticales d'un texte, mais on peut raisonnablement penser que les formations de niveau Bac+5 doivent pouvoir apporter une réponse.

\subsubsection{Opposition forme ing et forme simple}

49 Nous terminerons cet inventaire rapide et non exhaustif de points où l'explication de phénomènes langagiers intervenant dans la compréhension d'un texte passe par la grammaire récente par ce qui a longtemps constitué la première règle de grammaire anglaise vue en sixième, l'opposition forme en ing forme simple.

Le non-emploi de la forme en ing dans les indications scéniques appartient à l'histoire des explications proposées sur cette forme depuis 1975, en particulier par Henri Adamczewski ${ }^{19}$. Comme un débat récent ${ }^{20} l^{\prime}$ a montré, autant l'accord semble se faire sur le point de départ diachronique du marqueur et la multiplicité en langue actuelle des effets de sens, autant la nature de la présence de la catégorie grammaticale de l'aspect en anglais pose problème.

51 En lisant le scénario de The Big Lebowski, des étudiants se sont étonnés de la coexistence des règles pré- et post-adamczewskiennes ${ }^{21}$, à quelques lignes d'intervalle, dans des énoncés de contenu lexical similaire :

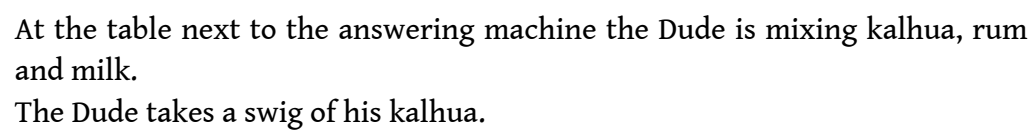

52 Il est inévitable, compte tenu des explications susceptibles d'être diffusées ici ou là, que des étudiants se posent la question de la durée relative des deux actions. La projection de la bande vidéo montre que cette manière de voir ne débouche sur rien. Par contre si on considère que mix renvoie à l'action qui se déroule sous les yeux du spectateur, et mixing à un commentaire cette action, on peut faire comprendre qu'il y a besoin de s'interroger sur l'implicite.

53 Au-delà des oppositions entre cadres théoriques ou manuels de grammaire, cet exemple montre que lorsque la compréhension est l'objectif prioritaire tout l'acquis de la recherche sur la langue est susceptible de rendre service. La sociologie de l'enseignement supérieur a pu constituer des barrières ici ou là, mais ce sont des passerelles qui sont nécessaires entre les enseignements de type DEA et ceux de type DESS.

\subsection{Terminologie}

54 Nous abordons en dernier cette question qui est vécue comme essentielle par beaucoup d'étudiants qui éprouvent un sentiment d'angoisse à se retrouver seuls sur un poste de travail, sans les camarades de promotion voire sans le professeur à qui demander conseil. 
Même si la sociologie récente du métier montre que le traducteur travaille de plus en plus dans une collectivité au sein d'un bureau de traduction et que le courrier électronique lui permette de contacter rapidement celui ou celle que la profession considère comme particulièrement compétent sur la question.

\section{Internet comme aide à la traduction des sigles}

Les sigles sont souvent le cauchemar des apprentis traducteurs et on peut penser qu'ils le seront encore plus pour les documentalistes et rédacteurs qui auront été moins formés à cet aspect de la néologie.

Si l'abondance des documents disponibles sur la toile multiplie inévitablement l'exposition à des sigles dont le lecteur n'a jamais entendu parler, elle paraît susceptible dans certains cas d'apporter la solution.

Dans notre corpus de science transdisciplinaire, au détour d'un article nous avons rencontré les sigles $v C J D, t C J D$ et $s p C J D$ dans un document émanant du centre d'Édimbourg qui suit l'épidémiologie de l'Encéphalite Spongiforme Bovine. Si CJD était connu, il paraissait aventureux de procéder par devinettes pour les minuscules. Quelques minutes de navigation, avec un outil dont le nom doit beaucoup à Jonathan Swift, ont suffi pour récupérer des textes où variant CJD, transmissible CJD et sporadic CJD apparaissaient en clair. Les gros dictionnaires ou les inventaires de sigles décodent des acronymes relativement fréquents comme MAFF (Ministry for Agriculture, Fisheries and Food) apparaissant dans la presse anglaise, USDA (United States Department of Agriculture) ou FCCC (Framework Convention on Climate Change) se manifestant dans la presse nord-américaine. On notera qu'avant la montée en puissance des moteurs de recherche, beaucoup de ces suites de lettres majuscules n'étaient décodables qu'après de longues lectures ou de coûteuses communications téléphoniques.

Une méthode similaire peut évidemment être suivie pour comprendre les néologies forgées par le chercheur au moment de sa découverte. De nombreux étudiants de quatrième et cinquième année se révèlent rapidement à l'aise sur ce type de recherche documentaire.

60 La même remarque s'applique aux néologies qui sont forgées dans les moments qui suivent la découverte d'un chercheur.

\subsection{Métaphores}

61 Nous avons abordé dans plusieurs publications antérieures cette question de la compréhension des métaphores qui constituent souvent des pièges redoutables y compris pour les étudiants les mieux adroits.

62 Il était prévisible qu'Internet n'échapperait pas à la tendance lourde qui fait de la métaphore une des matrices lexicogénétiques les plus productives. Si web et surf sont parfaitement identifiés, le transfert métaphorique de la terminologie de l'Internet vers la vie quotidienne est moins connu. Nous avons cité plus haut he's 404, nous trouvons aussi :

I bookmarked him after seeing his cool demo at Siggraph.

63 qui est intéressant dans la mesure ou le bookmark des internautes est lui même issu d'une métaphore. Autant le passage du marque-page à l'Internet a été facile en anglais autant le mécanisme paraît difficilement applicable au français. 

au défi que représente une multiplication, sans précédent dans l'histoire, de textes et documents présentant des réelles difficultés d'anglais dans les secteurs les plus développés de la vie économique et scientifique. linguistique, en terminologie et en grammaire, permettent de mettre en place dans des délais raisonnables une formation de nature à répondre à ce nouveau besoin social.

71 Les études anglaises disposent des outils pour adapter les recherches et l'enseignement menées en leur sein à l'insertion professionnelle de nombreux étudiants. A côté des grands débouchés traditionnels bien identifiés et bien maîtrisés que sont les préparations aux métiers de l'enseignement, il pourrait sembler de bonne méthode de développer des formations professionnelles diverses d'un établissement à l'autre, prenant appui sur les 
recherches qui y sont menées lorsque celles-ci correspondent à un besoin du marché de l'emploi.

\section{BIBLIOGRAPHIE}

Adamczewski, Henri et Jean-Pierre Gabilan. 1992. Les clefs de la grammaire anglaise. Paris : A. Colin. Adamczewski, Henri et Claude Delmas. 1982. Grammaire linguistique de l'anglais. Paris : Armand Colin.

Cotte, Pierre. 1996. L'Explication grammaticale de textes anglais. Paris : Presses Universitaires de France.

Delmas, Claude et al. 1993. Faits de langue en anglais. Paris : Dunod.

Delmas, Claude. 1987. Structuration abstraite et chaîne linéaire en anglais contemporain. Thèse. Paris : Cedel.

Hawkins, John. 1978. Definiteness and Indefiniteness. Londres : Croom Helm.

Lafont, Robert. 1978. Le Travail et la langue. Paris : Flammarion.

Joly, André et Dairine O'Kelly. 1009. Grammaire systématique de l'anglais. Paris : Nathan.

Souesmes, Jean-Claude. 1992. Grammaire anglaise en contexte. Gap : Ophrys.

Tournier, Jean. 1985. Introduction descriptive à la lexicogénétique de l'anglais contemporain. Genève : Champion Slatkine.

Vidalenc, Jean-Louis. 1997. «Quelques remarques sur l'emploi de la métaphore comme outil de dénomination dans un corpus d'histoire des sciences ». In Boisson, Claude et Philippe Thoiron (dir.), La dénomination. Lyon : Presses Universitaires de Lyon.

Vidalenc, Jean-Louis. 1998a. « Effet de came et métaphore, application d'un outil lexicogénétique à l'enseignement de la terminologie et de la syntaxe ». In Paulin, Catherine (dir.), Hommage à Jean Tournier. Dijon : Presses de l'Université de Franche Comté.

Vidalenc, Jean-Louis. 1998b. « Le subjonctif en anglais contemporain, extinction ou adaptation?». Journées d'études sur le subjonctif de l'URA CNRS 1030, Université Paris 4, septembre 1996. Modèles Linguistiques XIX/1, 127-142.

\section{NOTES}

1. Dans notre synthèse de travaux (voir bibliographie), nous prenons appui sur la notion de microspécialité (corpus constitué des articles et des livres figurant dans la bibliographie d'un DEA).

2. L'émoi que suscite l'introduction au CAPES d'une épreuve de compréhension d'une bande magnétique trois minutes est révélateur.

3. On trouve la réponse à ces questions et bien d'autres dans le site du projet aviator de l'Université de Liverpool (radar.rdues.liv.ac.uk). Le graphique de fréquences de zippergate figure 
sur un des transparents projetés lors de la conférence d'Antoinette Renouf à l'Université de Provence (dans le cadre du développement du volet linguistique des études de presse de l'Observatoire de la Société Britannique, 9 mars 1998).

4. Laurence Neuvecelle, Étude terminologique sur la fabrication du chapeau de feutre de poil, français anglais allemand, ISIT Paris 1992. (31 termes).

5. Jo de Jongh, Base de données de termes informatiques, IBM Belgique 1994 (14 000 termes).

6. Rappelons que nous avons travaillé en amont de nos quarante publications sur une dizaine de corpus conçus sur des bases différentes en fonctions d'objectifs complémentaires d'enseignement et de recherche. Le renouvellement périodique des sources nous semble important et nous regrettons une tendance à parfois figer la réflexion sur des sources mise en place il y a parfois trente ans et dont on connaît par ailleurs les limites.

7. Ce programme a été conçu pour l'Office of Scientific and Technical Information du Royaume-Uni. Une partie des documents et une erreur de cadrage du corpus de vulgarisation scientifique se retrouve dans The Sentence in Written English de Rodney Huddleston, Cambridge : CUP 1972.

8. On signalera que grâce à Claude Boisson, Pierre Busuttil et Jean-Louis Duchet, des liens permettent une connexion rapide entre les sites SAES et de nombreux journaux britanniques et américains.

9. C'est notre informatique portable et notre accès Internet personnel que nous utilisons à l'université de Provence.

10. Nous remercions pour ses informations sur le travail des pionniers Eugene Winter. Nous rappelons qu'il a participé avec Michael Halliday et Rodney Huddleston à la mise en place du corpus d'enseignement d'anglais scientifique du projet OSTI à l'Université de Londres en 1965.

11. Nous prévoyons un nouveau corpus de ce type, pour faciliter la compréhension par nos étudiants des écarts sociologiques avec leur vécu; nous envisageons une visite caméscopée du campus et des échanges d'informations entre étudiants par courrier électronique.

12. Traduisible par «il est à la masse ». L'origine de cette expression est le message d'erreur sur la toile : 404 URL not found.

13. Nous n'entrerons pas dans les détails, crédits de fonctionnement et crédits d'équipement, mais le travail informatique a été effectué hors de l'Université de Provence. Le corpus se présente sous forme d'une liste de serveurs où les scénarios sont disponibles.

14. Limitons nous à un exemple, pendant quelque mois le script de My Fair Lady a été accessible sur le serveur d'une université en Nouvelle Zélande.

15. D'après la société Yahoo France, le site le plus visité par les français au 1er semestre 1998 a été le scénario de Titanic.

16. Nous avons discuté de cette question dans Compréhension de l'anglais non standard, vidéo et formation initiale et permanente des enseignants, Colloque d'Avril sur l'anglais Oral Paris Nord 1992, Jean-Louis Duchet, John Humbley, Jean Michel Fournier \& Paul Larreya (dir.)., Paris APLV 1998. François Gallix a annoncé au congrès de la SAES de Nice (Mai 1997) la tenue d'une table ronde en Sorbonne autour de la traduction des romans de James Ellroy, considéré par bien des spécialistes du roman noir comme particulièrement difficile.

17. Nous pensons en particulier à une réaction vive de Leo Carruthers.

18. Nous avons traité de cette question du subjonctif dans Vidalenc 1(998b).

19. "Be+ing revisited", Eddy Roulet (dir.), New Insights in Applied Linguistics, Bruxelles : A IMAV 1975. Cette analyse, et le rôle des indications scéniques dans l'argumentation, a été développée plus tard dans sa thèse Be+ing en anglais contemporain et dans la grammaire linguistique de l'anglais.

20. Ce débat a été suscité par Claude Boisson sur la messagerie de l'ALAES : il a regretté que l'explication adamczewskienne, reprise par Jean-Pierre Gabilan, ne prenne pas suffisamment en compte la dimension diachronique. 
21. Avant 1975, en thème grammatical, une action en train de se faire appelait une forme en ing ; ensuite Adamczewski (1982) prend appui sur le fait que les indications scéniques sont au présent simple.

22. Rappelons que nous avons mentionné dans une publication antérieure avoir trouvé kiss of death dans un article de physique théorique.

\section{RÉSUMÉS}

Cet article propose un dispositif pédagogique de niveau Bac +5 permettant aux étudiants d'acquérir les bases linguistiques les rendant aptes à gérer la documentation multimédia d'une entreprise. L'analyse lexicale et grammaticale d'un panier de petits corpus de documents collectés sur Internet représente une base solide pour maîtriser la compréhension rapide de textes.

This paper discusses how corpora-based applied linguistics may help postgraduate students to acquire a working knowledge of English comprehension. The development of the Internet has convinced a number of firms that they need in-house personnel able to summarise - and translate if necessary - the pages they find of interest each day on the web. It is suggested that lexical and grammatical analysis of a network of small-size corpora may provide students with the relevant background knowledge.

\section{INDEX}

Mots-clés : enseignement de la compréhension (anglaise), enseignement des langues, linguistique appliquée, linguistique de corpus, nouvelles technologies

Keywords : applied linguistics, corpus linguistics, Internet, language teaching, teaching of (English) comprehension

\section{AUTEUR}

\section{JEAN-LOUIS VIDALENC}

Jean-Louis Vidalenc est professeur des universités à l'Université de Provence (Aix Marseille 1). Sa recherche porte sur la linguistique de corpus. Deux articles à paraître prendront appui sur un corpus électronique de soixante scénarios de films. Un portera sur un corpus Internet de communication semi médicale. jean-louis.vidalenc@orange.fr 